\title{
Differences in patient satisfaction with virtual telephone clinics in a tertiary referral centre for otolaryngology during and after lockdown measures during the SARS-Cov2 pandemic
}

\author{
Justin M. Hintze $\mathrm{e}^{1,2,3}$ - Isobel O'Riordan ${ }^{1,2,3} \cdot$ Tanvir Hussain $^{1} \cdot$ Emma Keane ${ }^{1,2,3} \cdot$ Paula Casserly ${ }^{1,3}$
}

Received: 27 October 2020 / Accepted: 16 December 2020 / Published online: 13 January 2021

(C) Royal Academy of Medicine in Ireland 2021

\begin{abstract}
Introduction The SARS-Cov2 pandemic has caused considerable disruption to provision of routine outpatient care. This pandemic has necessitated a more modern and innovative approach to clinics, which could potentially change outpatient organisation and improve efficiency in the long term. Telephone clinics are the most practical way to deliver healthcare at a distance.

Aim The purpose of the present study was to assess patient satisfaction with a virtual telephone clinic in a tertiary referral centre for otolaryngology first during the height of the SARS-Cov2 pandemic, and subsequently at a physical follow-up appointment after easing of "lockdown" measures.

Methods Patients were enrolled prospectively via a telephone interview over a 1-week period during the height of the "lockdown" measures, and subsequently at a physical appointment when measures eased.

Results Overall, patients responded very positively in the anonymised questionnaire at the time of their virtual appointment. However, at a subsequent physical appointment, there was a reduction in overall favourable responses from patients. While patients still thought virtual clinics were a good idea and convenient in the context of SARS-Cov2, we noted a reduction in satisfaction in other key aspects of their care. Notably, patients were less likely to think that virtual clinics were able to properly address their condition.

Conclusion While virtual clinics remain a useful tool during the height of lockdown measures during the SARS-Cov2 pandemic, we did note a significant reduction in favourable responses to virtual appointments over physical ones upon easing of lockdown measures. Patients remained cautious in suggesting that outpatient appointments may be replaced by virtual clinics.
\end{abstract}

Keywords Lockdown measures $\cdot$ SARS-Cov2 pandemic $\cdot$ Virtual clinics

\section{Introduction}

The COVID-19 pandemic has caused considerable disruption to provision of routine outpatient care. This is caused by a number of separate factors - patient concern in attending hospital for fear of exposure, healthcare worker illness and reduced staffing numbers, difficulty in travel and using public transport.

Justin M Hintze and Isobel O'Riordan are co-first authors

Justin M. Hintze

hintzej@tcd.ie

1 Department of Otolaryngology, Royal Victoria Eye and Ear Hospital, Dublin, Ireland

2 Royal College of Surgeons in Ireland, Dublin, Ireland

3 Trinity College Dublin, The University of Dublin, Dublin, Ireland
From the 13th of March, in anticipation of first peak of the SARS CoV-2 pandemic in Ireland, all elective surgery and outpatient clinics were cancelled. Patients were advised not to attend hospital unless in an emergency, and emergency department attendance fell by $45 \%$. [1] Surgical directorates within hospitals advised only life- or limb-saving surgery should be performed. This was at time of considerable heightened anxiety for both patients and healthcare workers, through the media images of patients laying on hospital floors in Spain and Italy that were shown, while health care professionals heard of the deaths of colleagues around the world. In the USA alone, between February and April 2020, 9200 healthcare workers contracted COVID-19. [2] ENT was considered to be a particularly high-risk group at this stage due to the high number of aerosol-generating procedures performed, not only in the operating theatre but also the considerable amount of flexible nasoendoscopy performed in the outpatient clinics. 
In Ireland, this crisis arrived in the context of already overburdened health care system and considerable waiting times for an ENT outpatient appointment [3]. Overcrowded outpatient waiting rooms, previously a daily occurrence, were no longer possible and stopped overnight. Waiting lists increased in size. Therefore, providing outpatient care for a large volume of patients during this pandemic was and continues to be a significant challenge.

Telephone clinics are the most practical way to deliver healthcare at a distance. They have been demonstrated to have high patient satisfaction rates across different disciplines, [4-6] yet are not routine practice in medicine in Ireland. In the initial wave of the SARS-CoV-2 pandemic, telephone consultations were used widely to triage patients and defer non-urgent appointments. [7] Following on from the initial peak, virtual clinics were used to assess both new and follow-up patients as required.

This pandemic has necessitated a more modern and innovative approach to clinics, which could potentially change outpatient organisation and improve efficiency in the long term.

Currently, a virtual clinic is safer for patients as it does not necessitate a face-to-face visit and therefore reduces potential COVID 19 contacts. While a direct cost reduction has not been identified in previous studies, [8] telephone consultations may reduce costs to society at large in terms of missed work days and the personal cost borne by patients.

Patient dissatisfaction at not attending their doctor face to face is often cited as a major concern when considering these clinics. We aimed to assess specifically patient experience with the virtual clinic within our ENT department.

\section{Aims and objectives}

The purpose of the present study was to assess patient satisfaction with a virtual telephone clinic in a tertiary referral centre for otolaryngology first during the height of the SARS-Cov2 pandemic, and subsequently at a physical follow-up appointment after easing of "lockdown" measures.

\section{Methods}

Virtual appointment Patients were enrolled prospectively via a telephone interview over a 1-week period during the height of the "lockdown" measures, with verbal consent obtained from each patient. Patients were identified from existing scheduled clinics. Patients were excluded if they did not consent to participation, complete the virtual telephone clinic follow-up, or did not complete the questionnaire. Thirty-two consecutive patients were enrolled, of which 2 either did not complete the questionnaire fully or did not attend for follow- up and were excluded. This left 30 patients available for final analysis.

Physical appointment To determine patient attitude towards virtual clinics following re-introduction of physical appointments, we surveyed 30 consecutive patients, enrolled prospectively from the same subspeciality otolaryngology clinic. Consent was obtained from each patient. Patients were excluded if they did not consent to participation or did not complete the questionnaire. Thirty patients consented for participation, of which 3 did not complete the questionnaire, and were excluded. This left 27 patients for final analysis.

For the virtual questionnaire, a 9-point questionnaire was devised (Table 1), with responses varying from 1 to 10 , with 10 indicating the most favourable response, and returned to the institution electronically and in an anonymised fashion.

For the physical questionnaire, a slightly amended 8-point questionnaire was used (Table 2). We also asked patients to provide their travel time to their physical appointment.

All data is presented as mean \pm standard deviation (SD), unless otherwise specified. All statistical analysis was performed using IBM® SPSS ${ }^{\circledR}$ ver 26 . To test differences between multiple groups, a one-way analysis of variance (ANOVA) was used, using post hoc Bonferroni correction to evaluate between-group differences.

\section{Results}

Of the 32 virtual patients enrolled prospectively during the study period, 30 patients completed their follow-up appointment and fully completed the brief virtual questionnaire.

Twenty-seven patients at a physical outpatient visit completed the questionnaire. The mean age was $46 \pm 16$, and $52 \%$ were female.

Table $1 \quad 9$-point virtual questionnaire

1. How would you rate your experience?

2. How would you rate the waiting time?

3. How would you rate the quality of the service provided?

4. How satisfied are you with the ability of virtual clinics to manage your condition?

5. How likely are you to recommend the service?

6. How would you rate the convenience of virtual clinics?

7. How likely are you to wish to have this service continue beyond the SARS-Cov2 pandemic?

8. How do you rate the clarity of the information given?

9. How confident are you with the service? 
Table 2 8-point physical questionnaire

1. Do you think virtual clinics are a good idea in the context of COVID-19?

2. How well do you think a virtual clinic would be able to address you condition?

3. How convenient do you think it is to have a virtual clinic?

4. How likely would you be to wish to have a virtual clinic over a physical appointment after the COVID-19 pandemic is over?

5. Do you think virtual clinic appointments are a sufficient replacement for physical appointments?

6. In the event of another "lockdown" scenario, how satisfied would you be with a virtual appointment over a physical one?

7. In the event of another "lockdown", how concerned would you be about attending hospital for your appointment?

8. How long does it take you to travel to the hospital for your outpatient appointment?

Virtual Overall, patients responded very positively in the anonymised questionnaire, with the most common response for every question being 10 (Table 3 ).

Patients responded, on average, most favourably to questions 1, 4 and 3 ("How would you rate your experience?", "How satisfied are you with the ability of virtual clinics to manage your condition?" and "How would you rate the quality of the service provided?" respectively), while the least favourable responses were for questions 8, 9 and 7 ("How do you rate the clarity of the information given?"; "How confident are you with the service?"; "How likely are you to wish to have this service continue beyond the SARS-Cov2 pandemic?" respectively).

A one-way ANOVA found a statistical difference between the questions $(p<0.001)$, with post hoc analysis finding a statistical difference between questions 7,8 and 9 , when compared with the remaining groups.

Table 3 Average Response to virtual questionnaire

\begin{tabular}{lll}
\hline Question Nr. & $\begin{array}{l}\text { Average response } \\
(\text { mean } \pm \text { SD) }\end{array}$ & $\begin{array}{l}\text { Most frequent response } \\
\text { (mode) }\end{array}$ \\
\hline 1 & $9.5 \pm 1.1$ & 10 \\
2 & $9.1 \pm 1.7$ & 10 \\
3 & $9.2 \pm 1.6$ & 10 \\
4 & $9.4 \pm 1.3$ & 10 \\
5 & $8.8 \pm 1.9$ & 10 \\
6 & $8.5 \pm 2.2$ & 10 \\
7 & $8.2 \pm 2.3$ & 10 \\
8 & $7.5 \pm 2.4$ & 10 \\
9 & $8.1 \pm 2.7$ & 10 \\
\hline
\end{tabular}

Physical During the physical questionnaire, we noted a reduction in overall favourable responses from patients (Table 4). While patients still thought virtual clinics were a good idea and convenient in the context of SARS-Cov2 (questions 1 and 2; mean response 7.4 and 7.1, respectively), we noted a reduction in satisfaction in other key aspects of their care. Notably, patients were less likely to think that virtual clinics were able to properly address their condition (question 2), with a reduction in response from 9.4 during the height of SARS-CoV-2 down to 4.9 at a physical appointment. Patients rated their willingness for a virtual appointment over a physical beyond the pandemic on average as a 5.5, whereas the average response for having a virtual appointment over a physical one "lockdown" measures that are re-introduced was 5.9. Overall, patients rated the ability of a virtual clinic to replace a physical clinic as only 4.9 (question 5).

Finally, the average time of patients spent travelling to their physical appointment was $60 \pm 32 \mathrm{~min}$.

\section{Discussion}

This study has demonstrated a high rate of patient satisfaction with telephone clinics in our institution during the pandemic. However, it also shows a reduction in satisfaction between patients at the height of SARS CoV-2 and patients following resumption of normal clinical activities.

During the peak of the pandemic, telephone consultations allowed us to stratify patients based on risk and also gave the patients an opportunity for contact or reassurance with their doctor. In essence, they acted as a stopgap between face-toface appointments. Our study has demonstrated a high satisfaction rate amongst patients in cases where outpatients visits may be high risk or inaccessible, but following on from the pandemic, patients felt their issues were better managed by face-to-face consultation. This may be explained as a "pandemic paradox". During the initial crisis, media coverage of hospitals struggling to adapt to changing circumstances led to

Table 4 Average response to physical questionnaire

\begin{tabular}{lll}
\hline Question Nr. & $\begin{array}{l}\text { Average response } \\
(\text { mean } \pm \text { SD) }\end{array}$ & $\begin{array}{l}\text { Most frequent response } \\
\text { (mode) }\end{array}$ \\
\hline 1 & $7.4 \pm 2.7$ & 10 \\
2 & $4.9 \pm 3.1$ & 1 \\
3 & $7.1 \pm 2.8$ & 10 \\
4 & $5.5 \pm 2.9$ & 5 \\
5 & $4.9 \pm 3.1$ & 5 \\
6 & $5.9 \pm 3.1$ & 10 \\
7 & $6.0 \pm 3.0$ & 5 \\
8 & $60 \pm 32(\mathrm{~min})$ & $\mathrm{Na}$ \\
\hline
\end{tabular}


a public outpouring of appreciation for "healthcare heroes". Restaurants delivered food to hospitals for healthcare workers; companies provided exclusive discounts. Public perception of healthcare workers at this stage was heroic, and patients felt grateful to have been contacted by a doctor under these circumstances. It may be that despite the reduction in routine services, public perception dramatically improved during the pandemic.

However, once routine care returned to normal and hospitals reinstated routine care, patients' frustrations at delayed appointments resurfaced quickly.

This study shows an interesting snapshot into two phases of public mood and hospital perception which may influence their experience of virtual clinics.

The data in the present study suggests a reduction in patient satisfaction with virtual clinics when lockdown measures ceased. This demonstrates a few important points about the importance of face-to-face consultations, not only in offering additional clinical cues, but also for overall patient satisfaction. Patients felt that a physical clinical appointment offers more reassurance regarding their symptoms, and that their needs were better met.

While our numbers are small, no patient in this cohort identified any areas of concern regarding the telephone clinics during the period of heightened restrictions. While previous studies have selected cohorts of patients deemed to be suitable for virtual consultations, we have included all patients scheduled for follow-up in our outpatients.

\section{Limitations of telephone clinics}

Telephone clinics have several limitations in their use. ENT is a procedure-based speciality - a significant portion of patients attending will have ear microsuctioning or flexible nasoendoscopy performed. This is obviously not possible via telephone consultation. Therefore, careful history taking and identification of concerning symptoms is essential. Furthermore, we found that in those cases, a physical appointment was nonetheless required, meaning that numerous follow-up reviews were required, leading to re-duplication of work.

Patients for whom English is not their first language may be disadvantaged in telephone clinics. Without the use of nonverbal cues which may aid understanding during face-to-face consultations, patients may struggle to understand over the phone.

Due to the nature of the speciality, many patients may be hearing impaired and again struggle without the use of lip reading and therefore not suitable for telephone clinics. However, it is important to note that these patients will be also disadvantaged by the use of masks in outpatient clinics, and we suggest identifying these patients in advance in order to avoid patient distress or frustration.
Future efforts for virtual clinics might include video consultations to help better assess patient symptoms.

\section{Travel time}

The average time spent travelling to an appointment was 60 min. This is a considerable burden, both in terms of actual time spent travelling and expense. This may indicate why, at certain times, patients who feel their condition is stable may wish to forgo their outpatient appointment.

\section{Conclusions}

From a healthcare planning perspective, we would suggest that there is a role going forward for telephone consultations in the provision of outpatient care. They are convenient for patients and time effective, and as we have demonstrated, have high levels of patient satisfaction. Patients, however, remained cautious in suggesting that outpatient appointments may be replaced by virtual clinics. This is likely to be multifactorial and not only based on the limitations of telephone consultations, but also on the intervention-based nature of otolaryngology clinics.

We would suggest that telephone clinics be a component of follow-up care and that they may be most suitable in patients with chronic rhinology or otology issues in order to maintain patient/physician contact and keep a link with the hospital to be reviewed at a physical appointment should issues arise. This will ultimately improve the accessibility of our clinics to patients, improve waiting times and also allow patients' greater flexibility in planning their follow-up care.

\section{Compliance with ethical standards}

Conflict of interest The authors declare that they have no conflict of interest.

\section{References}

1. Brick A, Walsh B, Keegan C, Lyons S COVID-19 and emergency department attendances in Irish public hospitals. [cited 2020 Oct 18]; Available from: https://doi.org/10.26504/qec2020may_SA_lyons

2. Burrer SL, de Perio MA, Hughes MM et al (2020) Characteristics of health care personnel with COVID-19 — United States, February 12-April 9, 2020. MMWR Morb Mortal Wkly Rep [Internet]. [cited 2020 Oct 18];69(15):477-81. Available from: https://pubmed.ncbi. nlm.nih.gov/32298247/. Accessed 27 Oct 2020

3. National Treatment Purchase Fund (NTPF) [Internet]. [cited 2020 Sep 13]. Available from: https://www.ntpf.ie/home/nwld.htm. Accessed 27 Oct 2020

4. Thompson JC, Cichowski SB, Rogers RG et al (2019) Outpatient visits versus telephone interviews for postoperative care: a randomized controlled trial. Int Urogynecol J [Internet]. [cited 2020 Sep 
13];30(10):1639-46. Available from: https://pubmed.ncbi.nlm.nih. gov/30783704/. Accessed 27 Oct 2020

5. Buchanan AH, Datta SK, Skinner CS et al (2015) Randomized trial of telegenetics vs. in-person cancer genetic counseling: Cost, Patient Satisfaction and Attendance. J Genet Couns [Internet]. [cited 2020 Sep 13];24(6):961-70. Available from: https://pubmed.ncbi.nlm.nih. gov/25833335/. Accessed 27 Oct 2020

6. Wang YC, Ganzorig B, Wu CC et al (2018) Patient satisfaction with dermatology teleconsultation by using MedX. Comput Methods Programs Biomed [Internet]. [cited 2020 Sep 13];167:37-42. Available from: https://pubmed.ncbi.nlm.nih.gov/30501858/. Accessed 27 Oct 2020

7. Paleri V, Hardman J, Tikka T et al (2020) Rapid implementation of an evidence-based remote triaging system for assessment of suspected referrals and patients with head and neck cancer on follow-up after treatment during the COVID-19 pandemic: model for international collaboration. In: Head and Neck [Internet]. John Wiley and Sons Inc.; [cited 2020 Sep 13]. p. 1674-80. Available from: https://pubmed.ncbi.nlm.nih.gov/32374942/. Accessed 27 Oct 2020

8. Dixon P, Beaver K, Williamson S et al (2018) Cost-consequence analysis alongside a randomised controlled trial of hospital versus telephone follow-up after treatment for endometrial cancer. Appl Health Econ Health Policy. [cited 2020 Sep 13];16(3):415-27. Available from: http://link.springer.com/10.1007/s40258-018-0378-6

Publisher's note Springer Nature remains neutral with regard to jurisdictional claims in published maps and institutional affiliations. 